\title{
Early Clinical Outcomes of Intrascleral Fixation Using an Intraocular Lens with Hook-Shaped Haptics
}

\section{Norihiko Yoshida' \\ Takashi Kojima (D) ${ }^{1,2}$ \\ Kazuo Ichikawa ${ }^{3}$}

'Department of Ophthalmology, Japanese Red Cross Gifu Hospital, Gifu, Japan; ${ }^{2}$ Department of Ophthalmology, Keio University School of Medicine, Tokyo, Japan; ${ }^{3}$ Chukyo Eye Clinic, Nagoya, Japan
Correspondence: Takashi Kojima Department of Ophthalmology, Keio University School of Medicine, Shinanomachi 35, Shinjuku-Ku, Tokyo, 160-8582, Japan

Tel +8I-3-5363-20I2

Fax+8I-3-5363-3087

Email tkojkoj@mac.com
Purpose: In this study, we aimed to report on the early results of intrascleral intraocular lens (IOL) fixation using a hook-shaped haptic IOL (hsh-IOL).

Patients and Methods: We enrolled 27 consecutive eyes of 27 patients (mean age, 74 \pm 11.4 years) who underwent intrascleral IOL fixation with an hsh-IOL and were followed-up for 3 months postoperatively. The reasons for surgery included insufficient capsular support, including IOL dislocation, aphakia, or dislocated crystalline lens. The haptic of the hsh-IOL was externalized from the eye using forceps, and the hook was buried in the scleral tunnel. We investigated the preoperative and 3-month postoperative corrected visual acuity (VA), intraocular pressure (IOP), corneal endothelial cell density, refractive prediction error, postoperative intraocular astigmatism, surgically induced astigmatism, and intraoperative and postoperative complications.

Results: The mean postoperative corrected VA $(\operatorname{logMAR}, 0.083 \pm 0.18)$ was significantly better than the mean preoperative value $(0.42 \pm 0.60, \mathrm{p}=0.0007)$. The 3 -month postoperative mean absolute prediction error was $1.00 \pm 0.96 \mathrm{D}$. The mean IOL-induced astigmatism was $0.95 \pm 0.70$ D. Further, the mean postoperative corneal endothelial cell count (2036 \pm 644 cells/ $\left.\mathrm{mm}^{2}\right)$ was significantly lower than the preoperative value $\left(2316 \pm 527 \mathrm{cells} / \mathrm{mm}^{2}\right)(\mathrm{p}=0.009)$. No patient had a 1-month postoperative IOP $<5 \mathrm{mmHg}$ or $>25 \mathrm{mmHg}$. There were no intraoperative or vision-threatening complications, such as retinal detachment, endophthalmitis, or IOL dislocation, due to postoperative haptics misalignment.

Conclusion: Intrascleral IOL fixation using hsh-IOL is an effective option for eyes with insufficient capsular support.

Keywords: intrascleral IOL fixation, efficacy, safety, hook-shaped haptics, visual acuity, intraocular lens

\section{Introduction}

In cases such as dislocation of crystalline lens, dropped nucleus during cataract surgery, or intraocular lens displacement, intraocular lens (IOL) fixation within the capsule is impossible. In such cases, IOL implantation methods include anterior chamber fixation, extracapsular fixation, suturing, and intrascleral fixation. Anterior chamber fixation has been reported to cause endothelial cell loss, ${ }^{1}$ and extracapsular fixation requires the presence of at least a portion of the capsule. Additionally, the complexity of IOL suturing technique can be problematic. To overcome the limitations of these methods, intrascleral fixation has recently become a widely used method for IOL implantation in eyes without capsular support. 
The intrascleral IOL fixation technique has been improved since it was first reported by Gabor SG and Agarwal et al. ${ }^{2-12}$ Currently, there are two major methods of scleral fixation: (i) the haptics of an existing IOL are grasped with forceps, externalized from the eye, and buried in the scleral tunnel, as represented by the Y-fixation technique (tunnel technique), ${ }^{7}$ or (ii) the haptics of an IOL are cauterized to create a flange and buried in the sclera, developed by Yamane et al (the flange technique). ${ }^{5,6}$ Although both techniques are appropriately sophisticated, the conventional three-piece IOL has been commonly used in both techniques.

We developed an IOL with U-shaped haptics (hook haptics), and first reported on an intrascleral IOL fixation technique using this lens in $2018 .{ }^{13}$ The lens was designed to facilitate easy grasping of the haptics with forceps during surgery, and to prevent the haptics falling into the vitreous cavity during surgery. Herein, we aimed to report on the early postoperative outcomes of intrascleral IOL fixation using a hook-shaped haptic IOL (hsh-IOL).

\section{Materials and Methods}

We retrospectively reviewed all patients who underwent intrascleral IOL fixation using the hsh-IOL (Xact NX70CH, Advance Vision Science Inc.) at our institution between June 6, 2017 and October 19, 2019. Only those patients who underwent postoperative follow-up examinations for 3 months were selected for the study. Our study was approved by the Institutional Review Board (IRB) of the Japanese Red Cross Gifu Hospital (Approval No. O17032801) and was conducted in accordance with the principles of the Declaration of Helsinki. Owing to its retrospective design, an opt-out method was approved by the IRB in place of written informed consent. The patients comprised 18 men and 9 women ( $\mathrm{N}=27$ eyes), with a mean age of $74.0 \pm 11.4$ years. Table 1 summarizes their demographic information.

The preoperative selection criterion for intrascleral IOL fixation using hsh-IOL at our institution was: patients aged $\geq 20$ years with insufficient capsular support, such as in aphakia, IOL dislocation, or crystalline lens dislocation.

\section{Intrascleral IOL Fixation Using Hsh-IOL}

The surgery was performed with some modifications to the previously reported method. ${ }^{13}$ Figure 1 shows the fixation method of the hsh-IOL into the sclera. In the previously reported method, a part of the hook-haptic was fixed next to the scleral incision. In the new method, the entire hook portion of haptic was buried in the scleral tunnel. Figure 2
Table I Patient Demographics

\begin{tabular}{|l|l|}
\hline Number of eyes & 27 \\
\hline $\begin{array}{l}\text { Age (years old, range) } \\
\text { Sex (Male/Female) }\end{array}$ & $\begin{array}{l}74.0 \pm \text { I I.4 (50-95) } \\
18 / 9\end{array}$ \\
\hline Preoperative lens condition (number of eyes) & \\
\hline IOL dislocation & 15 \\
\hline Dropped nucleus & 5 \\
\hline Aphakia & 6 \\
\hline Dislocated crystalline lens & 1 \\
\hline
\end{tabular}

Abbreviation: IOL, intraocular lens.

depicts the surgical procedure. The surgical video is provided in the Supplementary Video.

Following administration of the sub-Tenon's anesthetic block, we created a three-port vitrectomy setup and performed a 25-gauge vitrectomy using the Constellation ${ }^{\circledR}$ Vision system (Alcon). The dislocated IOL (if present) was removed. Using a 25 -gauge trocar knife at a tilt of $20^{\circ}$, a scleral incision was created at the 3 o'clock and 9 o'clock positions, $1.5 \mathrm{~mm}$ away from the limbus. We subsequently used a 23G MVR knife (MANI, Utsunomiya, Japan) to create an 2-mm long scleral tunnel in a counterclockwise direction from the scleral incision.

A corneal incision was then created at the 12 o'clock position using a $3.0-\mathrm{mm}$ slit knife. The hsh-IOL was inserted into the eye with an injector through the corneal incision; the leading haptic was placed in the vitreous space, and the trailing haptic was placed outside the eye. The latter was then grasped using the vitrectomy forceps and placed in the anterior chamber. We inserted the vitrectomy forceps through the scleral incision created at the 3 o'clock position. The hook portion of the trailing haptic was grasped at the level of the iris surface and externalized out of the eye. Vitrectomy forceps inserted through the scleral incision at the 9 o'clock position was used to grasp the hook portion of the leading haptic in the vitreous space and externalize it out of the eye. The hook portion of the haptic was subsequently buried in the scleral tunnel. Finally, we sutured the three sclerotomies for the vitrectomy ports and the scleral incisions at the 3 o'clock and 9 o'clock positions with an 8-0 absorbable thread.

\section{Primary Outcome Measures}

The primary outcome measures included the preoperative and 3-month postoperative corrected visual acuity (VA), 

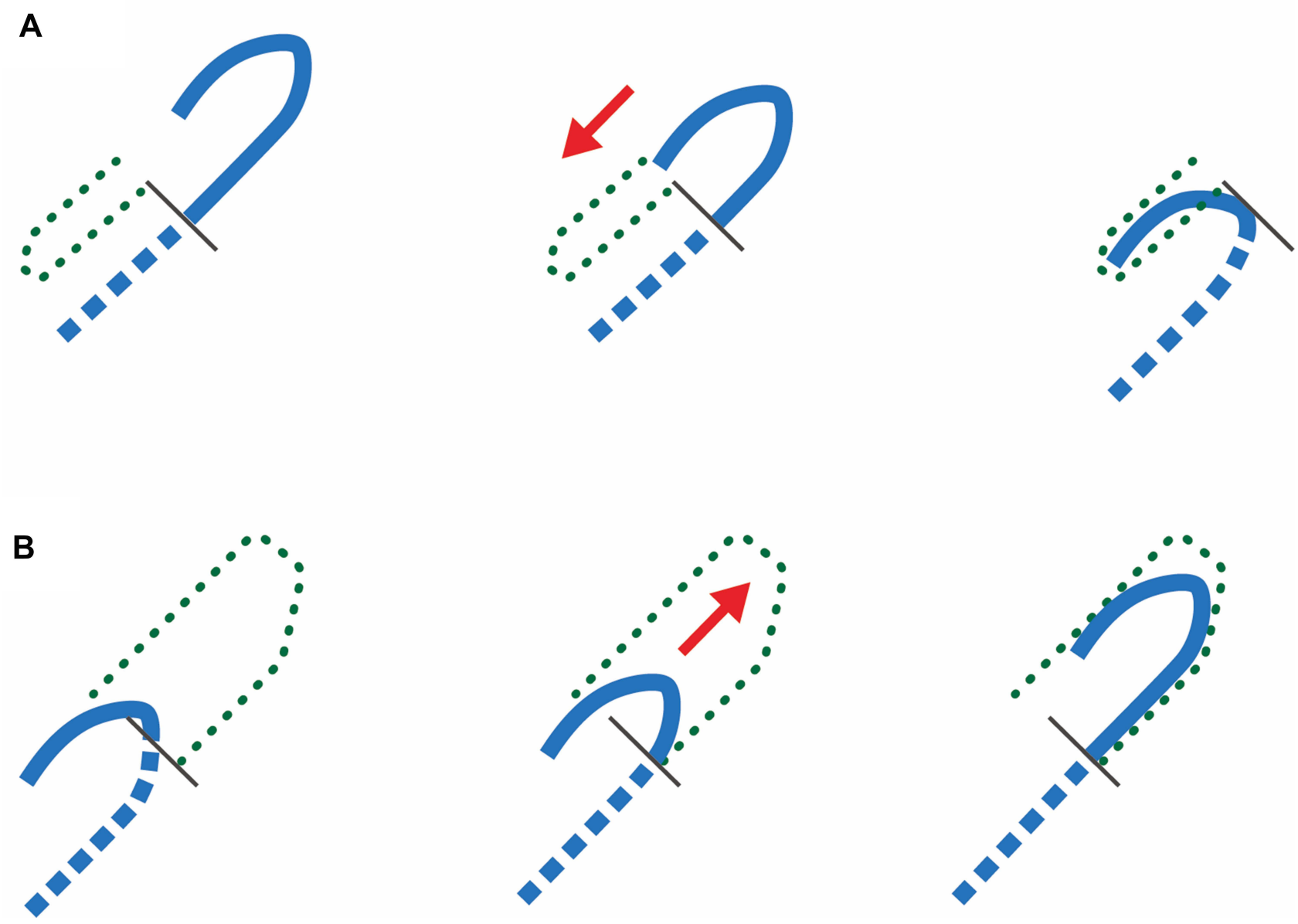

Figure I Surgical implantation of hook-shaped haptics into the sclera. (A) A previously reported method. The hook haptics have been implanted in the opposite direction of haptic withdrawal. (B) The improved method. The entire hook-shaped haptics have been buried in the direction in which they were pulled out. Arrow: the direction of implantation of hook haptics; dashed green line: the shape of scleral pocket; dashed blue line; IOL haptic inside the eye; solid blue line: IOL haptic; and solid black line: scleral wound.

intraocular pressure (IOP), corneal endothelial cell density, and refractive prediction error. We examined the VA using the Landolt ring chart and converted it to the logarithm of the minimum angle of resolution ( $\log M A R)$. The refractive prediction error was calculated by subtracting the predictive spherical equivalent from the manifested spherical equivalent, 3 months postoperatively. The corneal endothelial cell density was measured using a specular microscope (EM3000, TOMEY, Nagoya, Japan). All measurements were performed with auto-alignment; if this was not possible, the patient was asked to move their eyes slightly, and the average values were obtained in four directions.

\section{Secondary Outcome Measures}

We evaluated postoperative intraocular astigmatism by subtracting the value of postoperative corneal astigmatism from that of the total manifest astigmatism using the Jaffe vector analysis. ${ }^{14}$ Similarly, surgically induced corneal astigmatism was calculated using the Jaffe's vector analysis with the auto-keratometer values. Additionally, we assessed the intraoperative and postoperative complications.

\section{Hook-Shaped Haptic IOL}

The hsh-IOL is a modified version of the three-piece IOL (NX-70S), with a 7-mm optical diameter and haptics created from polyvinylidene fluoride. The length of the haptics was increased to $14 \mathrm{~mm}$, and the tip of the haptics had a 2-mm long and 1-mm wide U-shaped fold.

\section{Statistical Analyses}

GraphPad Prism (GraphPad software, Ver. 9.0, CA) was used for all statistical analyses. We performed the Wilcoxon signed-rank test to compare the preoperative and postoperative VA and corneal endothelial cell density. The Friedman test was performed to compare the changes in IOP over time, and the Dunn's multiple comparison test 

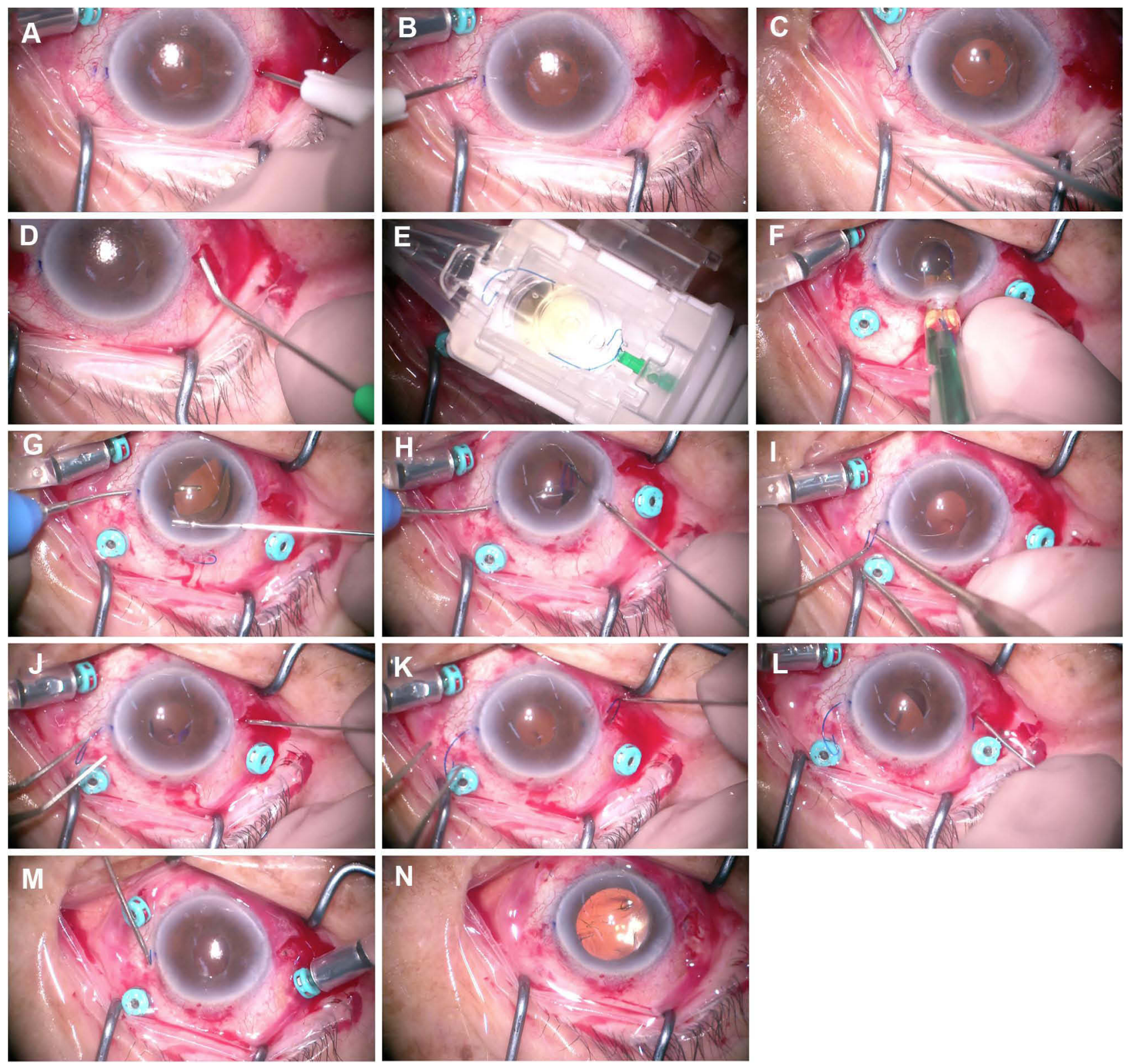

Figure 2 Modified intrascleral intraocular lens fixation surgery using a hook-shaped haptic intraocular lens. A scleral wound is created $180^{\circ}$ and $1.5 \mathrm{~mm}$ away from the corneal limbus, using the knife attached to the 25G trocar (A and B). A scleral tunnel is created with the 23G knife (C and D). The intraocular lens is loaded into the cartridge $(\mathbf{E})$ and inserted through the corneal incision $(\mathbf{F})$. Two forceps are used $(\mathbf{G}$ and $\mathbf{H})$ to grasp the trailing haptic and externalize it out of the eye (I). The other haptic is similarly grasped with the forceps $(\mathbf{J})$ and externalized out of the eye $(\mathbf{K})$. The hook-shaped part of the haptic is buried into the scleral tunnel $(\mathbf{L}$ and $\mathbf{M})$. Following trocar removal, the wound closure is confirmed, and the surgery is completed $(\mathbf{N})$.

was conducted as a post-hoc test. A p-value $<0.05$ was considered statistically significant.

\section{Results}

\section{Postoperative Visual Function}

Table 2 outlines the preoperative and postoperative corrected VA. The mean postoperative corrected VA was significantly better than the mean preoperative corrected VA $(0.083 \pm 0.176$ vs $0.419 \pm 0.601$, respectively; $\mathrm{p}=0.0007)$.

\section{Refractive Prediction Error}

The 3-month postoperative mean refractive prediction error and mean absolute refractive prediction error were $-0.30 \pm 1.31 \mathrm{D}$ and $1.00 \pm 0.96 \mathrm{D}$, respectively.

\section{IOL-Induced Astigmatism}

To evaluate IOL-induced astigmatism, we subtracted the value of the postoperative corneal astigmatism from that of the postoperative manifest astigmatism using vector 
Table 2 Preoperative and 3-Month Postoperative Visual Acuity and Other Examination Values

\begin{tabular}{|l|l|l|l|}
\hline & Preoperative & $\begin{array}{l}\text { 3-Months } \\
\text { Postoperatively }\end{array}$ & P-value \\
\hline $\begin{array}{l}\text { DCVA (logMAR) } \\
\text { IOP }(\mathrm{mmHg})\end{array}$ & $0.419 \pm 0.601$ & $0.083 \pm 0.176$ & 0.0058 \\
$\begin{array}{l}\text { Corneal } \\
\text { endothelial cell } \\
\text { density }\left(/ \mathrm{mm}^{2}\right)\end{array}$ & $2316 \pm 527$ & $13.0 \pm 3.1$ & 0.79 \\
\hline
\end{tabular}

Abbreviations: logMAR, logarithm of minimum angle resolution; DCVA, distance corrected visual acuity; IOP, intraocular pressure.

analysis. The mean IOL-induced astigmatism was 0.95 $\pm 0.70 \mathrm{D}$. In contrast, the surgically induced corneal astigmatism was $1.14 \pm 0.80 \mathrm{D}$.

\section{Corneal Endothelial Cell Density}

Among the 27 eyes included in this study, we assessed the postoperative corneal endothelial cell density in 23 eyes with available preoperative measurements. The postoperative corneal endothelial cell density was significantly lower than the preoperative corneal endothelial cell density $\left(2036 \pm 644\right.$ cells $/ \mathrm{mm}^{2}$ vs $2316 \pm 527$ cells $/ \mathrm{mm}^{2}$, respectively) $(\mathrm{p}=0.009)$.

\section{Intraocular Pressure}

The mean preoperative IOP was $16.6 \pm 6.5 \mathrm{mmHg}$. Six eyes had a preoperative IOP $\geq 22 \mathrm{mmHg}$. These six cases included: (i) a patient referral due to an incomplete surgery owing to a posterior capsule rupture (parts of the nucleus were retained in the vitreous at the time of the visit [preoperative IOP $=36 \mathrm{mmHg}$ ); (ii) a patient referral due to IOL insertion failure owing to the weak zonules (the patient had vitreous hemorrhage [preoperative IOP $=27$ $\mathrm{mmHg}]$ ); (iii) a dislocated IOL and Soemmering's ring in the vitreous (preoperative IOP $=25 \mathrm{mmHg}$ ); (iv) a torn IOL during cataract surgery (preoperative IOP $=23.3$ $\mathrm{mmHg}$ ), a patient with aphakia at 1 week postoperatively (IOP=23.3 mmHg); (v) a case of dropped nucleus (preoperative IOP $=22.7 \mathrm{mmHg}$ ); and (vi) a dislocated IOL (preoperative IOP $=22.4 \mathrm{mmHg}$ ).

The mean IOPs at 1 day, 1 week, 1 month, and 3 months postoperatively were $10.3 \pm 5.8 \mathrm{mmHg}, 13.2 \pm 4.5$ $\mathrm{mmHg}, 13.1 \pm 3.1 \mathrm{mmHg}$, and $13.1 \pm 3.1 \mathrm{mmHg}$, respectively. The 1-day postoperative IOP was significantly lower than the preoperative IOP $(\mathrm{p}<0.001)[<5 \mathrm{mmHg}$ in $7 / 27$ eyes $(26 \%)$, and $>21 \mathrm{mmHg}$ in $1 / 27$ eye $(3.7 \%, 25$
$\mathrm{mmHg})]$. There were no cases with 1-month postoperative IOP $<5 \mathrm{mmHg}$ or $>25 \mathrm{mmHg}$.

\section{Complications}

Among the 27 eyes, $8(30 \%)$ had mild postoperative vitreous hemorrhage, which resolved 1 week later. Iris capture occurred in one eye, which was corrected by a repositioning surgery 2 days postoperatively; it has not recurred since. There were no postoperative cases of haptics falling into the vitreous cavity or protruding out of the eye, which could have resulted in IOL dislocation. There were no cases of retinal detachment or cystoid macular edema. Moreover, we did not observe other complications that might affect the visual function.

\section{Discussion}

To perform intrascleral IOL fixation safely, the haptics should be easily graspable within the eye, easy to fixate, and should remain stable in the sclera. Therefore, we developed an hsh-IOL that was shaped like the grip of a walking stick, which facilitated its grasp using forceps and reduced the risk of the IOL falling into the vitreous cavity during surgery. Following its burial in the sclera, the haptics would supposedly be difficult to dislocate and would maintain a stable position. Herein, we investigated the early postoperative results of intrascleral IOL fixation using hsh-IOL and evaluated its efficacy and safety.

In our previous report, we grasped a hook-shaped haptic using a customized hook with a ring-shaped tip, and externalized it out of the eye. ${ }^{13}$ Despite their benefits, customized hooks are often unavailable. Thus, we modified the technique to accommodate the use of easily available conventional vitrectomy forceps. In a previous report, ${ }^{13}$ after externalizing the hook-shaped haptic out of the eye, a tunnel was created next to the scleral incision, and only a part of the hook haptic was buried in the tunnel. However, the length of the haptics implanting in the sclera could not be adjusted using the aforementioned method, and therefore it was difficult to adjust the position of the IOL in some cases. Thus, we adopted a technique in which the entire hook portion of the hook-shaped haptic was buried in a tunnel.

In this study, the postoperative refractive prediction error was $-0.30 \pm 1.31 \mathrm{D}$, with an absolute mean of 1.00 \pm 0.96 D. Previously reported results for Yamane's doubleneedle technique have demonstrated a mean refractive prediction error of $-0.21 \pm 0.99 \mathrm{D}^{6}{ }^{6}$ Further, Mitamura et al have reported the results of intrascleral IOL fixation 
using an endoscope to confirm needle insertion into the ciliary sulcus, in which the absolute mean of the refractive prediction error was $0.86 \pm 0.59 \mathrm{D}^{4}$ Our findings were comparable to those in the previously reported studies.

However, a previous study comparing intrascleral fixation with cataract surgery using the same type of in-thebag IOL implantation technique reported that the mean absolute prediction error of intrascleral fixation was greater than that of intracapsular implantation. ${ }^{15}$ Once the hook haptics of hsh-IOL are buried in the sclera, the fixation becomes stable However, it is difficult to move and adjust the position of the haptics. This warrants the need for future studies to develop a technique to further reduce the predicted refractive error.

In this study, the scleral incision was created $1.5 \mathrm{~mm}$ from the corneal limbus; however, previous reports indicate that the scleral incision are created $2 \mathrm{~mm}$ from the corneal limbus. ${ }^{5-7}$ In the first 10 cases in this study, the position of the scleral incision was confirmed intraocularly using an ophthalmic endoscope during the surgery to ensure that it was close to the original position of the IOL fixation in the capsule. However, the fact that the average predicted refractive error was $-0.3 \mathrm{D}$, which is slightly myopic, might have been related to the position of the scleral incision. Further research is needed to examine the adjustment of the scleral incision position in a larger number of cases.

The IOL-induced intraocular astigmatism was 0.95 $\pm 0.70 \mathrm{D}$. Intraocular astigmatism may be caused by the tilt or decentration of the IOLs. With the flange method, a misalignment in the positions of the two cauterized flanges, or a difference in the angle of needle penetration or in the tunnel length affects the tilt of the IOL directly. In contrast, with the tunnel method, several factors such as misalignment of the two scleral incisions, different lengths of the buried haptics, or large scleral pockets could increase the IOL tilt. Additionally, in a typical threepiece IOL, the haptics are tilted $5^{\circ}-10^{\circ}$ toward the posterior capsule from the optic to prevent iris capture. The haptic of the IOL used in this study was angled at $7^{\circ}$. Therefore, the IOL may be more prone to tilting if one haptic is pulled more than the other. If the haptics were not tilted in relation to the optic, they would not tilt while being pulled horizontally. This, in turn, would be effective in preventing IOL-induced astigmatism. Improvements in IOL and surgical techniques are required to prevent intraocular astigmatism.
A previous study reported an average IOL tilt of $5.21^{\circ}$ $\pm 1.47^{\circ}$ during in-the-bag IOL implantation in conventional cataract surgery. ${ }^{15}$ However, we were unable to evaluate the postoperative IOL tilt due to the unavailability of anterior segment optical coherence tomography. An IOL tilt may affect visual function by causing coma aberration and astigmatism, thereby necessitating an evaluation of the amount of IOL tilt and an improvement in the technique to achieve a tilt similar to that of in-the-bag IOL implantation cases. To reduce the IOL tilt while performing our technique, it is important to ensure that the two scleral incisions are performed in symmetrical positions, the scleral tunnel has a constant tilt, and the haptic length is evenly drawn into the scleral tunnel. It may also be useful to use an endoscope to ensure that the position of the haptic inserted into the eye is similar to that reported previously. $^{3}$

In previous studies, $0-5.3 \%$ cases of low postoperative IOP were observed with the flange method, ${ }^{4,6,8}$ compared to $0-21.4 \%$ cases observed with the tunnel method. ${ }^{16-20}$ In this study, $26 \%$ ( $7 / 27$ eyes) eyes had an IOP $<6 \mathrm{mmHg}$ during the early postoperative period, and the percentage tended to be higher than that of the previously reported data. In general, the flange method has a smaller incision for externalizing the haptics than the tunnel method. The flange method may be superior in wound closure and may result in a stable postoperative IOP. Moreover, in one study, no cases of low postoperative IOP were observed with the tunnel method. ${ }^{17}$ Hence, the skill of the surgeon and the method of suturing the scleral wound may be influential factors. Additionally, among the seven eyes with low IOP, the existing IOL was removed in two eyes. The scleral corneal incision in these eyes was created at the 12 o'clock position, which was close to the corneal incision that had been created in the previous cataract surgery. In such situations, the wounds may overlap in some cases, leading to insufficient closure. The low IOP during the early postoperative period suggested a leakage of aqueous humor from the incision site, thereby suggesting the need to improve the surgical technique to prevent serious complications such as endophthalmitis.

In this study, the rate of vitreous hemorrhage was 30\% (8/ 27 eyes), which was higher than the previously reported rate of $5 \%$ observed with the flange method. ${ }^{6}$ The vitreous hemorrhage was likely due to low IOP caused by bleeding from the scleral incision at the site of the vitrectomy port. Thus, in the tunnel method, it is necessary to meticulously confirm that there is no leakage at the end of the surgery. Moreover, the 
incision size can be reduced by using the L-shaped incision ${ }^{21}$ for IOL removal, which prevents the likelihood of low postoperative IOP, vitreous hemorrhage, and infection.

Furthermore, in this study, we observed one case of iris capture immediately after surgery, which did not recur following the repositioning surgery. There were no postoperative cases of haptics getting exposed outside the eye or falling inside the vitreous cavity, which could have resulted in IOL dislocation. Despite secure fixation of the IOL being an advantage of the hsh-IOL, a previous report showing haptics exposure 4 months postoperatively ${ }^{22}$ necessitates a long-term follow-up. Intrascleral IOL fixation is associated with potential vision-threatening complications, including retinal detachment and endophthalmitis. However, no vision-threatening complications were observed in this study, and we confirmed the short-term safety of the surgery.

This study has several limitations. Its retrospective design may have introduced patient selection bias. Additionally, we only evaluated the clinical outcomes of intrascleral fixation using the hsh-IOL. Future randomized studies should compare the process using other techniques. Further, this study was performed at a single institution and by a single surgeon. This warrants an evaluation of the postoperative results by multiple surgeons at various institutions in the future. Furthermore, the hsh-IOL may be useful for inexperienced surgeons owing to the easy externalization of the haptics out of the eye. Future studies should determine the surgical outcomes with inexperienced surgeons.

\section{Conclusion}

Intrascleral fixation with hsh-IOL was shown to be an effective surgical treatment, with no vision threatening complications; however, there was a tendency toward development of early postoperative complications such as low IOP and vitreous hemorrhage.

\section{Ethics Approval and Informed Consent}

Our study was approved by the Institutional Review Board (IRB) of the Gifu Red Cross Hospital (Approval No. O17032801) and was conducted in accordance with the principles of the Declaration of Helsinki. Owing to its retrospective design, an opt-out method was approved by the IRB in place of written informed consent.

\section{Acknowledgments}

We thank Mr. Seiji Tokiwa for editing the surgery video.

\section{Author Contributions}

Conception (Yoshida N., Kojima T., Ichikawa K.). Study design (Yoshida N., Kojima T.). Execution of study (Yoshida N., Kojima T.). Acquisition of data (Yoshida N.). Analysis and interpretation (Yoshida N., Kojima T., Ichikawa K.). Writing the article (Yoshida N., Kojima T., Ichikawa K.). Revising and critically reviewed the article ((Yoshida N., Kojima T., Ichikawa K.). All authors have agreed on the journal to which the article will be submitted. All authors reviewed and agreed on all versions of the article before submission. All authors agreed to take responsibility and be accountable for the contents of the article.

\section{Funding}

This study was self-funded.

\section{Disclosure}

Dr Norihiko Yoshida reports personal fees from Santen Pharmaceutical. Dr Norihiko Yoshida and Dr Kazuo Ichikawa are the inventors and report a patent for the hook-shaped haptic intraocular lens licensed to Chukyo Medical. Professor Takashi Kojima reports personal fees from Alcon Japan, Santen Pharmaceutical, Otsuka Pharmaceutical, and STAAR Surgical, outside the submitted work. Dr Kazuo Ichikawa reports grants from Alcon, HOYA, personal fees from ZEISS, STAAR, Santen, NIDEK, KY CenterVue, holds copyright for Igaku shoin, personal fees/grants for lectures from Kowa, JFC, Novartis Pharma, and MANI, outside the submitted work. The authors declare that they have no other competing interests.

\section{References}

1. Sawada T, Kimura W, Kimura T, et al. Long-term follow-up of primary anterior chamber intraocular lens implantation. $J$ Cataract Refract Surg. 1998;24(11):1515-1520. doi:10.1016/S0886-3350(98) 80176-4

2. Agarwal A, Kumar DA, Jacob S, et al. Fibrin glue-assisted sutureless posterior chamber intraocular lens implantation in eyes with deficient posterior capsules. J Cataract Refract Surg. 2008;34(9):1433-1438. doi:10.1016/j.jcrs.2008.04.040

3. Gabor SGB, Pavlidis MM. Sutureless intrascleral posterior chamber intraocular lens fixation. $J$ Cataract Refract Surg. 2007;33 (11):1851-1854. doi:10.1016/j.jcrs.2007.07.013

4. Mitamura H, Kaga T, Kojima T, et al. Clinical outcomes of endoscope-assisted 30-gauge single-needle technique for intrascleral intraocular lens fixation. Ophthalmic Res. 2021;64(2):253-260. doi: $10.1159 / 000511047$ 
5. Yamane S, Inoue M, Arakawa A, et al. Sutureless 27-gauge needle-guided intrascleral intraocular lens implantation with lamellar scleral dissection. Ophthalmology. 2014;121(1):61-66. doi:10.1016/j. ophtha.2013.08.043

6. Yamane S, Sato S, Maruyama-Inoue M, et al. Flanged intrascleral intraocular lens fixation with double-needle technique. Ophthalmology. 2017;124(8):1136-1142. doi:10.1016/j. ophtha.2017.03.036

7. Ohta T, Toshida H, Murakami A. Simplified and safe method of sutureless intrascleral posterior chamber intraocular lens fixation: y-fixation technique. J Cataract Refract Surg. 2014;40(1):2-7. doi:10.1016/j.jcrs.2013.11.003

8. Kim S, Kim JT, Špiritović M. The simply modified intrascleral fixation using round flange (SMURF) technique for intrascleral intraocular lens fixation. Sci Rep. 2021;11(1):1-9. doi:10.1038/ s41598-020-79139-8

9. Tamburrelli C, Tamburrelli AC. Sutureless scleral fixated IOL: the "Catcher Pole" technique. Clin Ophthalmol. 2021;15:121-128. doi:10.2147/OPTH.S288769

10. Samir A, ElHag YG, Elsayed AMA, et al. Scleral fixation of single-piece foldable IOL using double-flanged technique. Clin Ophthalmol. 2020;14:3131-3136. doi:10.2147/OPTH.S276226

11. Agarwal R, Sidhu N, Sinha R, et al. Real-time optical coherence tomography-guided scleral flap creation for intrascleral haptic fixation of posterior chamber intraocular lens. Clin Exp Ophthalmol. 2020;48(9):1310-1312. doi:10.1111/ceo.13862

12. Zong Y, Wu K, Fang W, et al. Modified intrascleral fixation for repositioning the dislocated single-piece, rigid PMMA intraocular lens. Retina. 2020. doi:10.1097/IAE.0000000000002876

13. Yoshida N, Kojima T, Yokoyama S, et al. New surgical approach for intrascleral fixation using an intraocular lens with hook-shaped haptics. J Cataract Refract Surg. 2018;44(2):129-133. doi:10.1016/ j.jcrs.2017.12.011

14. Kojima T, Kaga T, Watanabe M, et al. Clinical evaluation of the arched blade for cataract surgery. Acta Ophthalmol Scand. 2005;83 (3):306-311. doi:10.1111/j.1600-0420.2005.00419.x
15. Torii T, Tamaoki A, Kojima T, et al. Comparison of clinical outcomes between intracapsular implantation and intrascleral fixation using the same model of intraocular lens. Clin Ophthalmol. 2020;14:3965-3974. doi:10.2147/OPTH.S268126

16. Chantarasorn Y, Techalertsuwan S, Siripanthong P, et al. Reinforced scleral fixation of foldable intraocular lens by double sutures: comparison with intrascleral intraocular lens fixation. Jpn J Ophthalmol. 2018;62(3):365-372. doi:10.1007/s10384-018-0579-4

17. Kumar DA, Agarwal A, Packiyalakshmi S, et al. Complications and visual outcomes after glued foldable intraocular lens implantation in eyes with inadequate capsules. J Cataract Refract Surg. 2013;39 (8):1211-1218. doi:10.1016/j.jcrs.2013.03.004

18. Yang Y, Yao TT, Zhou YL, et al. A modified intrascleral intraocular lens fixation technique with fewer anterior segment manipulations: 27-gauge needle-guided procedure with built-in 8-0 absorbable sutures. BMC Ophthalmol. 2019;19(1):1-9. doi:10.1186/s12886019-1239-2

19. Zhao H, Wang $\mathrm{W}, \mathrm{Hu} \mathrm{Z}$, et al. Long-term outcome of scleral-fixated intraocular lens implantation without conjunctival peritomies and sclerotomy in ocular trauma patients. BMC Ophthalmol. 2019;19 (1):1-9. doi:10.1186/s12886-019-1172-4

20. Nb K, Kohli P, Pangtey BPS, et al. Evaluation of sutureless, glueless, flapless, intrascleral fixated posterior chamber intraocular lens in children with ectopia lentis. J Ophthalmol. 2018;2018:3212740. doi:10.1155/2018/3212740

21. Hennekes RL, Van den Dooren KA. Asymmetric L-shaped corneal no-stitch tunnel incisions for cataract surgery. $J$ Cataract Refract Surg. 1999;25(4):550-555. doi:10.1016/S0886-3350(99)80054-6

22. Matsui Y, Matsubara H, Hanemoto T, et al. Exposure of haptic of posterior chamber intraocular lens after sutureless intrascleral fixation. BMC Ophthalmol. 2015;15(1):1-3. doi:10.1186/s12886015-0102-3
Clinical Ophthalmology

\section{Publish your work in this journal}

Clinical Ophthalmology is an international, peer-reviewed journal covering all subspecialties within ophthalmology. Key topics include: Optometry; Visual science; Pharmacology and drug therapy in eye diseases; Basic Sciences; Primary and Secondary eye care; Patient Safety and Quality of Care Improvements. This journal is indexed on PubMed

\section{Dovepress}

Central and CAS, and is the official journal of The Society of Clinical Ophthalmology (SCO). The manuscript management system is completely online and includes a very quick and fair peer-review system, which is all easy to use. Visit http://www.dovepress.com/ testimonials.php to read real quotes from published authors. 